\title{
Hemodialysis Catheter-related Infections: Incidence, Microbiology and Outcome 5 years of Dubai Hospital Experience
}

\author{
Hussein Yousif Salem, Maseer Ahmed, Kashif Gulzar, Fakhriya Alalawi, and Amna Alhadari
}

\section{ABSTRACT}

Background: The central venous catheters (CVC) has recently become a worldwide commonest technique for vascular access, however; prone to infection and associated with other complications more than other vascular access techniques, such as fistula or graft.

Objective: To investigate the incidence, the types of micro-organism, the most sensitive and suitable antibiotics, the outcomes associated with CVC infection in chronic hemodialysis patients treated through tunneled CVC.in a single-center population.

Methods: The records of hemodialysis patients from January 2012 to December 2016 were reviewed. Patients above 14 years of age, on hemodialysis via a permanent tunneled catheter at any time of the mentioned period, were included. The rate of CRBSI (Catheter-Related Blood Stream Infections) was calculated as density of incidence and reported per 1000 catheter days. Patient mortality was recorded.

Results: In 5 years, a total of 73 episodes of CRBSI in 49 hemodialysis patients were recorded. $59.2 \%$ were male with a mean age of 53.57 years. $\mathbf{4 0 . 8 \%}$ had end-stage kidney disease secondary to diabetic nephropathy. In all, 67.3\% episodes grew Gram-positive isolates, among those staphylococci epidermidis $(26.4 \%, \mathrm{n}=19)$ and staphylococci aureus $(25 \%$, $\mathrm{n}=18$ ) were the most common organism, whereas Klebsiella pneumonia $(8.3 \%, n=6)$, Enterobacter cloacae $(8.3 \%, n=6)$, pseudomonas aeruginosa $(5.5 \%, n=4)$ and E.coli $(5.5 \%, n=4)$ were the most common Gram-negative isolates. A total of 6 isolates were multidrug-resistant, which includes Klebsiella pneumonia, Citrobacter, and E. coli. Only 1 out of 18 staphylococci aureus cases were methicillin-resistant and $21 \%(n=6)$ of Gram-negative isolates were Extended-spectrum beta-lactamase (ESBL) producing organisms. The average hospital stay was 9 days (2-30 days). The hemodialysis catheter was removed for $19(38.77 \%)$ patients and 4 (8.1\%) patients expired due to septic shock despite catheter removal and being on appropriate antibiotics.

Conclusion: The mainstay to reduce CRBSI would be ideal to avoid catheters or decrease the duration of catheters. This can be achieved through more aggressive counseling and fast-track protocols for AV Fistulae creation at the pre-dialysis stage.

Keywords: Catheter-Related Blood Stream Infections (CRBSIs), Central Venous Catheters (CVC), End-Stage Renal Disease, Hemodialysis.
Published Online: July 10, 2021

ISSN: $2736-5476$

DOI: $10.24018 /$ ejclinicmed.2021.2.3.97

Hussein Yousif Salem

Department of Nephrology, Dubai Hospital, Dubai Health Authority, Dubai, United Arab Emirates.

Maseer Ahmed

Department of Nephrology, Dubai Hospital, Dubai Health Authority, Dubai, United Arab Emirates.

Kashif Gulzar

Department of Nephrology, Dubai

Hospital, Dubai Health Authority, Dubai, United Arab Emirates.

Fakhriya Alalawi*

Department of Nephrology, Dubai Hospital, Dubai Health Authority, Dubai, United Arab Emirates.

(e-mail: fjalalawi08@yahoo.co.uk)

Amna Alhadari

Department of Nephrology, Dubai Hospital, Dubai Health Authority, Dubai, United Arab Emirates.

\section{*Corresponding Author}

\section{INTRODUCTION}

With the availability of a range of advanced permanent vascular access techniques for hemodialysis [from arteriovenous fistula (AVF) to an arteriovenous graft (AVG) and the central venous catheters (CVC)]; there has been an increase in the reported incidence of morbidity and mortality directly attributed to these, particularly with the use of CVC [1]-[3]. National Kidney Foundation Kidney Disease
Outcome Qualitative Initiative (NKF KDOQI) recommends planning hemodialysis vascular access creation on reaching chronic kidney disease stage IV [4], despite that CVCs are still used in about one-third of all the hemodialysis units and has become a worldwide commonest technique for vascular access [5], [6]. Refusals of acceptance of dialysis, unable to create vascular access timely, and maturation failure of AVF in time are some of the reasons for the prevalence of $\mathrm{CVC}$ in the dialysis population. Hemodialysis vascular catheters 
could be temporary, inserted mostly in the internal jugular or femoral vein and last for 2 weeks or tunnel cuffed catheters left for few months. Nevertheless, vascular catheters are associated with an increased risk of infection, thrombosis, need for rescue procedure, and increased hospitalization and mortality than AVF [7]. Catheters are responsible for half of the infections in hemodialysis patients, and Catheter-related bloodstream infections (CRBSIs) are the second most common cause of death, also a major cause of catheter loss and metastatic infection in hemodialysis population[8]-[10]. Its incidence is a minimum of 0.6 to a maximum of 6.5 episodes per 1000 catheter-days [7], [8], [11], [12]. As compared to native AVF, the estimated risk of infection for temporary and tunnel catheters is 25.5 and 15.5 , respectively [13]. Staphylococcus is a causative organism in most cases, however; infections may also be caused by other grampositive and gram-negative pathogens. Multiple postulations can explain the pathogenesis of CRBSI, which includes colonization of the catheter tip, cutaneous track, and formation of biofilm inside the catheter lumen as well as on the external surface that may also spread to the bloodstream [14]. Diabetes mellitus, peripheral vascular disease, patient age, and the site of catheter infection are potential risk factors for CRBSI. There was an absolute dearth of data on this critical issue of care of ESRD patients in the local context. The current retrospective study was therefore conducted to assess the incidence, risk factors associated with catheter infections and types of micro-organism, the most sensitive and suitable antibiotics, the common outcomes associated with CVC infection, and patient characteristics in ESRD patients in a single-center population.

\section{METHOD}

This is retrospective study was conducted in the Dialysis unit of Dubai hospital from 1st January 2012 to 31 st December 2016. The study population was end-stage kidney disease patients above 14 years of age, who were receiving hemodialysis via a permanent catheter. Patients whose blood culture did not yield any growth or yield isolate in the periphery but not in ports were excluded. The study was approved by a scientific research review board of the Dubai Health Authority. Patients' clinical and demographic data were collected from the hospital system in particular information about vascular catheters and CRBSI. Under sterile conditions, an interventional radiologist inserts a permanent hemodialysis catheter wearing gloves, masks, and gowns. No antibiotic prophylaxis was used. Catheter insertion was avoided in cases of bacteremia, and sepsis was treated with antibiotics according to culture \& sensitivity. Semi occlusive dressing was used to cover catheters and the exit site was swabbed with povidone prior to use during inpatient and sometimes antibiotic or ferrous injections were also given via catheter. Heparin is used for locking catheters to maintain patency on completion of hemodialysis, provided there is no contraindication. TauroLock as a catheter lock solution was installed in the majority of cases as well. The diagnosis of CRBSI is made when there is a blood culture positive at least from the peripheral vein and one of the catheter ports. On suspecting CRBSI, blood cultures were taken from 2- catheter ports and peripheral veins and according to our dialysis unit protocol and patient will receive empirical vancomycin and ciprofloxacin to cover grampositive and gram-negative organisms. Further management depends upon patient blood culture results, clinical condition, and response to antibiotics.

The rate of CRBSI was calculated as density of incidence and reported per 1000 catheter days. Patient mortality was recorded. The results are expressed as numerical values and percentages for categorical variables and mean \pm standard deviation (SD) for continuous variables.

\section{RESULTS}

In a span of 5 years, 49 patients suffered catheter-related infections, $59.2 \%(n=29)$ were male and their mean age was 53.57 years. The most common ethnicity was Emirati $61.2 \%$ $(n=30)$. Overall, $40.8 \%(n=20)$ had end-stage kidney disease secondary to diabetic nephropathy, whereas primary glomerulonephritis, hypertension, chronic allograft nephropathy and obstructive uropathy accounted for $22.4 \%$ $(n=11), 14.3 \% \quad(n=7), 8.1 \% \quad(n=4) 6.1 \% \quad(n=3)$ of cases, respectively. A large section of our study population also suffers from other comorbidities, which include hypertension $(53.4 \%)$, diabetes mellitus $(30.13 \%)$, and ischemic heart disease $(18.4 \%)$, (Table I). There were 73 total episodes of CRBSI in 49 hemodialysis patients during the study period, the incidence of CRBSI was 1.7 episodes per 1000 catheter days and total catheter days were 44976. Fifteen different organisms were isolated and only three patients had two or more bacteria isolates. In all, 67.3\% $(n=49)$ CRBSI episodes were caused by Gram-positive isolates, among those staphylococci epidermidis $(26.4 \%, \mathrm{n}=19)$ and staphylococci aureus $(25 \%, \mathrm{n}=18)$ were the most common organism, whereas Klebsiella pneumonia $(8.3 \%, \mathrm{n}=6)$, Enterobacter cloacae $(8.3 \%, \mathrm{n}=6)$, pseudomonas aeruginosa $(5.5 \%, \mathrm{n}=4)$ and E.coli $(5.5 \%, \mathrm{n}=4)$ were most common Gram-negative isolates (table-2). In CRBSI patients, $68 \%$ of Staphylococcus epidermidis infections were resistant to the usual antibiotics such as penicillin G, oxacillin, cephalosporin, and clindamycin, and appeared sensitive only to vancomycin $(84 \%)$, teicoplanin $(68.4 \%)$, and linezolid (52.6\%). Equally Staphylococcus hominis and Staphylococcus haemolyticus had a similar resistant pattern. Also, the sensitivity of staphylococci aureus isolates was $100 \%$, and $72.2 \%$ for vancomycin and oxacillin respectively, while Enterobacter cloacae were $100 \%$ sensitive to ciprofloxacin (table-3). A total of 6 isolates were multidrug-resistant, that includes two each of Klebsiella pneumonia, Citrobacter, and E.coli. Only 1 out of 18 staphylococci aureus cases were methicillinresistant and $21 \%(n=6)$ of Gram-negative isolates were Extended-spectrum beta-lactamase (ESBL) producing organisms. The average duration of stay in the hospital was $9.20 \pm 5.11$ days, also the hemodialysis catheter was removed for $19(38.77 \%)$ patients and $4(8.1 \%)$ patients expired due to septic shock despite catheter removal and being on appropriate antibiotics. 
TABLE I: PATIENT'S CHARACTERISTICS

\begin{tabular}{lc}
\hline \multicolumn{1}{c}{ Number of CRBSI Patients } & $\mathrm{n}=49$ \\
\hline Age & $53.57(10.9)$ \\
Gender & 29 male $(59.2 \%)$ \\
Cause of End-stage Kidney Disease & \\
Diabetes Mellitus & $20(40.8 \%)$ \\
Chronic Glomerulonephritis & $11(22.4 \%)$ \\
Hypertension & $7(14.3 \%)$ \\
Rejected Transplant & $4(8.1 \%)$ \\
Obstructive Uropathy & $3(6.1 \%)$ \\
Others & $4(8.1 \%)$ \\
Comorbidities & \\
Hypertension & $39(53.4 \%)$ \\
Diabetes Mellitus & $22(30.1 \%)$ \\
Ischemic Heart Disease & $13(17.8 \%)$ \\
Mortality during the study period & $4(8.1 \%)$ \\
\hline
\end{tabular}

TABLE II: NUMBER AND PERCENTAGE OF ORGANISMS ISOLATED FROM BLOOD CULTURE IN CASES OF CRBSI

\begin{tabular}{clcc}
\hline \multirow{2}{*}{ Bacteria } & \multicolumn{1}{c}{ Name of Organism } & $\begin{array}{c}\text { No. of } \\
\text { isolates }\end{array}$ & $\%$ \\
\hline Gram +ve & Staphylococcus Epidermidis & 19 & $26.40 \%$ \\
& Staphylococcs Aureus & 18 & $25 \%$ \\
& Staphylococcus Capitis & 2 & $2.77 \%$ \\
& Staphylococcus Hemolyticus & 2 & $2.77 \%$ \\
& Staphylococcus Warneri & 2 & $2.77 \%$ \\
& Staphylococcus Hominis & 2 & $2.77 \%$ \\
& Enterococcus Fecailis & 2 & $2.77 \%$ \\
Gram -ve & Enterococcus Fecum & 2 & $2.77 \%$ \\
& Kleibsiella Pneumonia & 6 & $8.30 \%$ \\
& Enterobacter spp & 6 & $8.30 \%$ \\
& Pseudomonas Aeruginosa & 4 & $5.50 \%$ \\
& Escherichia Coli & 4 & $5.50 \%$ \\
& Citrobacter spp & 2 & $2.77 \%$ \\
& Pseudomonas Maltophilia & 1 & $1.38 \%$ \\
& Candida spp & 1 & $1.38 \%$ \\
\hline
\end{tabular}

TABLE III: BACTERIAL SENSITIVITY PROFILE

\begin{tabular}{lcccc}
\multicolumn{5}{c}{ TABLE III: BACTERIAL SENSITIVITY PROFILE } \\
& $\begin{array}{c}\text { Gram }+\boldsymbol{v} \boldsymbol{e} \\
\text { Staphylo- } \\
\text { coccus } \\
\text { Epidermidis }\end{array}$ & $\begin{array}{c}\text { Staphylo- } \\
\text { coccus } \\
\text { Aureus }\end{array}$ & $\begin{array}{c}\text { Klebsiella } \\
\text { pneumonia }\end{array}$ & $\begin{array}{c}\text { Entero- } \\
\text { bacter } \\
\text { cloacae }\end{array}$ \\
\hline Vancomycin & $84.2 \%$ & $100 \%$ & & \\
Clindamycin & $21 \%$ & $33.3 \%$ & & \\
Linezolid & $77 \%$ & $11.1 \%$ & & \\
Teicoplanin & $68.4 \%$ & $22.2 \%$ & & \\
Cephalosporin & $15.7 \%$ & $44.4 \%$ & & \\
Oxacillin & & $83.3 \%$ & & \\
Ciprofloxacin & & & $66.6 \%$ & \\
Cefuroxime & & & $50 \%$ & \\
Gentamicin & & & $16.6 \%$ & 0 \\
Levofloxacin & & & 0 & $50 \%$ \\
Meropenem & & & & \\
\hline
\end{tabular}

\section{DISCUSSION}

Hemodialysis catheters are unavoidable for many dialysis patients during their dialysis history, some may need it for initiation of hemodialysis in the absence of functioning arterio-venous fistula (AVF) or graft (AVG) and others may require it as a temporary measure when complications arise from AVF/AVG render them non-functional. One of the commonest complications of Hemodialysis catheter is Infection, Catheter-related bloodstream infection (CRBSI) is the most significant manifestation. Type of catheter, diagnostic method, patient population are some of the variables, determine the reported rate of CRBSI. Studies have reported an incidence rate of 0.46 to $30 \%$ per 1000 catheter days and 4.3 to $26 \%$ of inserted catheters [11], [12]. We observed an incidence rate of CRBSI in our population of 1.7 per 1000 catheter days or 7-8\% per year. In the Arabic world, the Incidence of CRBSI up to $23.6 \%$ per year in Saudi and 6 cases per 1000 catheter days in Egypt [15], [16]. In our study, we observed that a longer duration of the catheter was a risk factor for catheter infection. Diabetes mellitus and peripheral vascular disease were identified by other studies as significant risk factors. The diagnosis of CRBSI in our study was based on clinical signs, symptoms, and positive blood culture from one catheter lumen and peripheral vein. The infectious disease society of America (IDSA) and the center for disease control (CDC) publish their extensive guidelines for the diagnosis of CRBSI [17]. This requires isolation of the same pathogen from a blood culture drawn through the central line and simultaneously from a peripheral vein with a single bacterial colony count, at least threefold higher in the central line sample compared to that obtained from a peripheral vein for a definite diagnosis of CRBSI [18]. Similarly, the new NHS diagnosis of CRBSI is done by any of the following 2 criteria; the same organism isolated from peripheral blood culture and from quantitative ( $>15$ colonyforming units) culture of the catheter tip or the same organism isolated from a peripheral and a catheter lumen blood culture [19]. The new CDC definitions require a presence of clinical manifestations and at least 1 positive blood culture from a peripheral vein (with no other apparent source), with either positive semiquantitative (>15 CFU/catheter segment) or quantitative (>102 CFU/catheter segment) culture, of the same organism isolated from the catheter segment [20]. The latest KDOQI-2019 guidelines have almost similar CDC definitions for diagnosing CRBSI [21]. There are other organism-specific molecular methods using PCR to diagnose methicillin-resistant staphylococcus aureus associated with CRBSI, but it is of high cost and not available widely. Different complications of CRBSI for example Infective endocarditis, spinal abscesses, septic arthritis, and septic emboli must be diagnosed and managed aggressively. In our institute, we used to treat suspected CRBSI with vancomycin and ciprofloxacin after taking blood cultures (from both Dialysis catheter ports and peripheral vein), the final decision of antibiotic relies on culture and sensitivity. In addition, our priority is to salvage the catheter as possible. Removal of Catheter is the last resort in case of severe sepsis or recurrent CRBSI to give patient line free break, followed by insertion of non-tunneled or tunneled catheter when bacteremia resolves. Nevertheless, we do remove the permanent catheters in all cases of staph aureus, pseudomonas sepsis, and candida positive cultures, regardless of response to therapy. According to the recommendation by the International Society of Nephrology, Chronic kidney disease patients at stage IV should be counseled for renal replacement therapy [22], for that purpose, the idea of renal school was introduced in our center, where a team of dialysis nurses and doctors inform patient and family about the importance of vascular access and renal replacement therapy with the help of audiovisual aids, also vascular access clinic is working in liaison with dialysis unit to facilitate early vascular access creation. Despite these efforts, our ratio of dialysis patients with permanent catheters is quite high because many patients are unwilling to accept prophylactic vascular access creation due to cultural social stigmas unless they suffer complications of ESRD like acute pulmonary edema and urgent dialysis provided. Despite pre-dialysis counseling, the rate of 
initiation of hemodialysis from AVF is quite below the international goals [23]. AVF use for initiation of hemodialysis is observed in $44 \%$ and $40 \%$ of patients in the U.K and Europe respectively [24]-[26], this number has further dropped to $18 \%$ and $10 \%$ in U.S.A and Singapore. In our study population, $66 \%$ of hemodialysis patients had AVF/AVG in 2012, this percentage increase to $72 \%$ in 2016. Regarding the range of bacteria implicated in CRBSI, Grampositive isolates caused most infections $(n=49,68.05 \%)$, compared to $24(32 \%)$ episodes caused by a gram-negative organism, our study findings are supported by a significant number of literature [27]-[29], while studies from India and Singapore reported more cases of CRBSI due to Gramnegative isolates [30], [31]. Moreover, there is common resistance in our population towards penicillin $\mathrm{G}$, oxacillin, Augmentin, cephalosporins, tetracycline, clindamycin, and levofloxacin, and the resistance was shown mostly by the $\mathrm{S}$. epidermidis, Staphylococcus hominis, Staphylococcus haemolyticus, and Enterococcus faecalis. More local studies are needed to determine whether the bacteriologic profile of tunneled catheters is different from other parts of the world and the source of infection.

Our study has certain limitations. First of all; being a retrospective study, it could not remove any potential bias. Second, Blood cultures were collected only for suspected cases so we may have missed subclinical bacteremia episodes, also vancomycin resistance was not determined because only one isolate of CRBSI was methicillin-resistant. Moreover, our study did not take account of antibiotics in patients who might have taken antibiotics prior to treatment. Yet; it is the first study of its kind in the local context and has provided valuable evidence regarding the risk of bacteremia in hemodialysis patients and local microbiology which is of utmost importance for recommending appropriate empirical antimicrobial treatment.

\section{CONCLUSION}

With limited study, we can conclude that the mainstay to reduce CRBSI would be ideally to avoid catheters or decrease the duration of catheters. So an early creation of AVF in the pre-ESRD patients can reduce patient morbidity and mortality. This can be achieved through more aggressive counseling and fast-track protocols for AVF creation.

\section{AUTHOR CONTRIBUTIONS}

All authors contributed extensively to the work presented in this paper.

\section{FUNDING}

The authors have no relevant financial support.

\section{COMPLIANCE WITH ETHICAL STANDARDS}

Conflict of interest: All authors have declared no competing interest.
Ethical Approval: The study was approved by the scientific research committee of the Dubai Health Authority.

\section{REFERENCES}

[1] Agarwal AK, Haddad NJ, Vachharajani TJ, Asif A. Innovations in vascular access for hemodialysis. Kidney Int. 2019 May;95(5):105363. doi: 10.1016/j.kint.2018.11.046.

[2] Arhuidese IJ, Orandi BJ, Nejim B, Malas M. Utilization, patency, and complications associated with vascular access for hemodialysis in the United States. J Vasc Surg. 2018 Oct; 68(4):1166-74.

[3] Allon M. Vascular access for hemodialysis patients: new data should guide decision making. Clin J Am Soc Nephrol. 2019 Jun 7;14(6):954961. doi: 10.2215/CJN.00490119.

[4] Rodríguez-Aranda A, Alcazar JM, Sanz F, et al. Endoluminal colonization as a risk factor for coagulase-negative staphylococcal catheter-related bloodstream infections in hemodialysis patients Nephrol Dial Transplant 2011; 26(3): 948-955.

[5] Gallieni M, Giordano A, Rossi U, Cariati M. Optimization of dialysis catheter function. J Vasc Access. 2016 Mar; 17 Suppl 1:S42-6.

[6] Bohlke M, Uliano G, Barcellos FC. Hemodialysis catheter-related infection: prophylaxis, diagnosis and treatment. J Vasc Access. 2015 Sep-Oct;16(5):347-55.

[7] McCann M, Einarsdottir H, Van Waeleghem JP, et al. Vascular access management III: central venous catheters. J Ren Care 2010; 36(1): 2533.

[8] Dittmer ID, Sharp D, McNulty CA, et al. A prospective study of central venous hemodialysis catheter colonization and peripheral bacteremia. Clin Nephrol 1999; 51: 34-39.

[9] Johnson WJ, Kurtz SB, Mitchell JC, et al. Results of treatment of hemodialysis center patients. Mayo Clin Proc 1984; 59: 669-671.

[10] Kovalik EC, Raymond JR, Albers FJ, et al. A clustering of epidural abscesses in chronic hemodialysis patients: risks of salvaging access catheters in cases of infection. J Am Soc Nephrol 1996; 7: 2264-2267.

[11] Kaur M, Gupta V, Gombar S, Chander J, Sahoo T. Incidence, risk factors, microbiology of venous catheter associated bloodstream infections-a prospective study from a tertiary care hospital. Indian J Med Microbiol. 2015 Apr-Jun;33(2):248-54.

[12] Napalkov P, Felici DM, Chu LK, et al. Incidence of catheter-related complications in patients with central venous or hemodialysis catheters: a health care claims database analysis. BMC Cardiovasc Disord 2013; 13: 86.

[13] Taylor G, Gravel D, Johnston L, et al. Prospective surveillance for primary bloodstream infections occurring in Canadian hemodialysis units. Infect Control Hosp Epidemiol 2002; 23: 716-720.

[14] Gahlot R, Nigam C, Kumar V, et al. Catheter-related bloodstream infections. Int J Crit Illn Inj Sci 2014; 4(2): 162-167.

[15] Ibrahim M, Fahad R.A, Sumaiya I, et al. Hemodialysis catheter-related infections: Result of tertiary care center study in Saudi Arabia. Int J Med Sci Public Health 2019;8(4): 318-25.

[16] Amira M, Malik H.A, Khaled A.M, et al. Incidence of Central line associated blood stream infection in intensive care unit in private hospital (Cairo, Egypt). Open Publ Health J 2018; 11: 562-71.

[17] Leonard A.M, Michael A, Emilio B, et al. Clinical practice guidelines for diagnosis and management of intravascular catheter related infection: 2009 update by infectious disease society of America. Clinical infectious disease 2009; 49(1): (1-45).

[18] Haddadin Y, Annamaraju P, Regunath H. Central line associated blood stream infections (CLABSI). StatPearls [Internet]. 2020 Jun 22. Accessed on Jan 2021.

[19] https://www.dbth.nhs.uk/wp-content/uploads/2017/12/CRBSIfinal.pdf. Accessed on Jan 2021.

[20] https://www.cdc.gov/infectioncontrol/pdf/guidelines/bsi-guidelinesH.pdf Accessed on Jan 2021.

[21] Lok CE, Huber TS, Lee T, Shenoy S, Yevzlin AS, Abreo K, Allon M, Asif A, Astor BC, Glickman MH, Graham J. KDOQI clinical practice guideline for vascular access: 2019 update. American Journal of Kidney Diseases. 2020 Apr 1;75(4):S1-64

[22] Bolton WK. Renal Physicians Association Clinical Practice Guideline: Appropriate Patient Preparation for Renal Replacement Therapy: Guideline Number 3. Journal of the American Society of Nephrology. 2003 May 1;14(5):1406-10.

[23] Vassalotti JA, Jennings WC, Beathard GA, Neumann M, Caponi S, Fox $\mathrm{CH}$, et al. Fistula first breakthrough initiative: targeting catheter last in fistula first. Semin Dial. 2012;25(3):303-10.

[24] de Pinho NA, Coscas R, Metzger M, Labeeuw M, Ayav C, Jacquelinet C, Massy ZA, Stengel B. Vascular access conversion and patient outcome after hemodialysis initiation with a nonfunctional 
arteriovenous access: a prospective registry-based study. BMC nephrology. 2017 Dec;18(1):1-1.

[25] Malas MB, Canner JK, Hicks CW, Arhuidese IJ, Zarkowsky DS, Qazi $\mathrm{U}$, et al. Trends in incident hemodialysis access and mortality. JAMA Surg. 2015;150(5):441-8.

[26] Noordzij M, Jager KJ, van der Veer SN, Kramar R, Collart F, Heaf JG, et al. Use of vascular access for haemodialysis in Europe: a report from the ERA-EDTA Registry. Nephrol Dial Transplant. 2014;29(10):1956-64.

[27] Allon M. Dialysis catheter-related bacteraemia: treatment and prophylaxis. Am J Kidney Dis 2004;44(5):779-91.

[28] Lok CE, Mokrzycki MH. Prevention and management of catheterrelated infection in haemodialysis patients. Kidney international. 2011;79(6):587-98.

[29] Betjes MG. Prevention of catheter-related bloodstream infection in patients on haemodialysis. Nature reviews. Nephrology. 2011;7(5):257-65.

[30] Shefali G, Shrikara P.M, Ashok B, Shrikala B. Microbiology of Nontunneled catheter related infections. 2016;10(7):24-8.

[31] Hao Y.Y, Shu C.P, Chei S.T, et al. Catheter related complications and survival among incident hemodialysis patients in Singapore. J Vasc Access. 201819(6):602-08. 